\title{
Focal parenchymal sparing in fatty infiltration of the liver
}

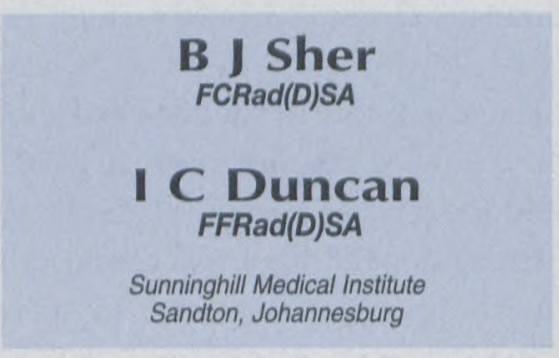

\section{Introduction}

Fatty infiltration within the liver usually shows a diffuse pattern throughout the organ. However, focal areas of either normal liver tissue sparing or fatty infiltration can occur and can be confused with metastatic disease on sectional imaging, unless one is aware of the typical sites and imaging appearances of these variants.

\section{Case report}

A 40-year-old obese woman presented with intermittent right-sided abdominal pain. Ultrasound examination of the liver showed a solitary well-defined focal echolucent lesion in the region of the caudate lobe just anterior to the common hepatic duct and portal vein, measuring $2 \mathrm{~cm}$ in diameter. The rest of the hepatic parenchyma showed increased echogenicity throughout suggesting diffuse fatty infiltration (Fig. 1).

A computed tomography (CT) scan showed decreased attenuation

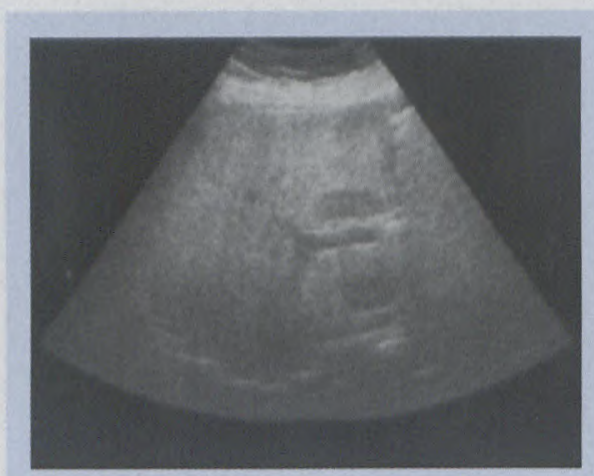

Fig. 1. Axial abdominal ultrasound showing diffuse echogenicity of the liver parenchyna with a hypoechoic 'lesion' near the porta hepatis.

throughout most of the parenchyma again suggesting fatty infiltration, but with areas of normal attenuation seen at the liver periphery. Furthermore, in the same area of the caudate lobe a discrete rounded area with the same diameter as that measured on the earlier ultrasound examination was noted. This area showed a higher attenuation than the surrounding parenchyma, similar to the areas around the periphery of the liver (Fig. 2). This area showed enhancement during the arterial, portal return and delayed phases of a triphasic contrastenhanced liver scan compatible with that of normal liver tissue, whereas the rest of the fat-infiltrated parenchyma showed a lesser degree of enhancement during all three phases (Figs 3 and 4).

On the basis of the ultrasound and CT findings, together with previous

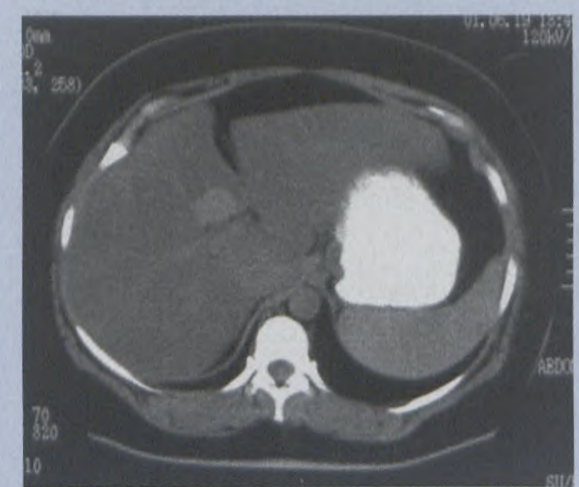

Fig. 2. Non-contrast CT scan of the abdomen showing the typical hypodense appearance of a fat-infiltrated liver, with peripheral sparing and the rounded area near the porta hepatis representing focal sparing. It is important not to confuse this round area of fatty sparing with a neoplasm.

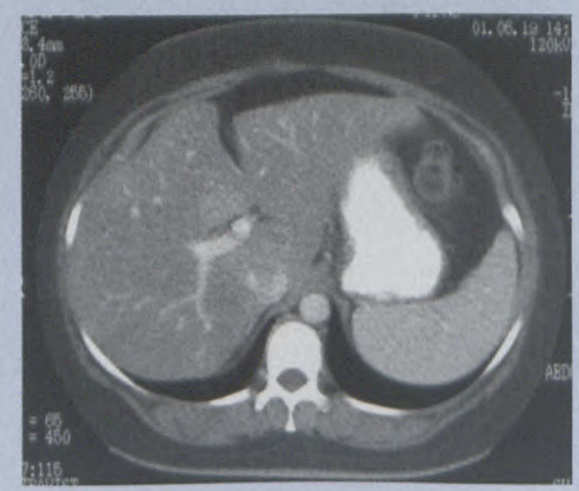

Fig. 3. Post-contrast abdominal CT scan during the portal venous return phase showing that the fatinfiltrated liver enhances less than the area of sparing seen in Fig. 2.

ultrasound reports which indicated no change in the size or appearance of the focus over a period of several months, it was felt that the focal 'lesion' most probably represented an area of focal fatty sparing. The findings were felt to be pathognomonic enough in this particular case so that the added expense of MRI for confirmation was unwarranted. The spleen was also found to be enlarged (Fig. 4).

\section{Discussion}

The usual causes of fatty infiltration of the liver are alcohol abuse, obesity, malnutrition, chemotherapy, hyperalimentation, diabetes, steroid 


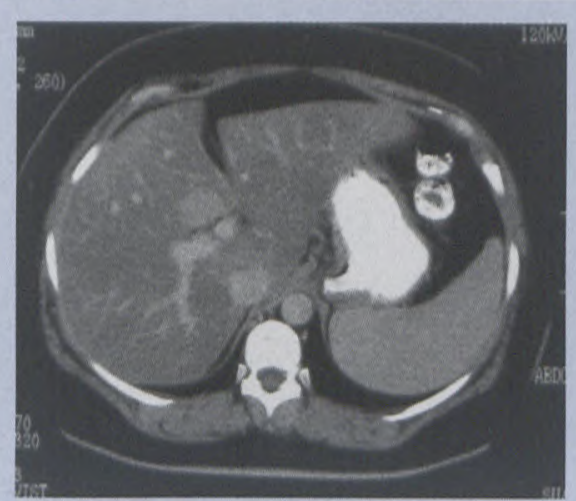

Fig. 4. Post-contrast CT scan of the abdomen delayed phase, showing a bulky spleen. This is typ ically seen in a non-alcoholic fatty liver.

administration, Cushing's syndrome and radiation hepatitis.

Despite a more typical pattern of diffuse parenchymal infiltration, residual foci of normal unaffected liver parenchyma can be present, usually at the periphery of the liver or abutting the porta hepatis and gallbladder fossa. These may be mistaken for neoplastic lesions unless one is aware of the patterns of fatty sparing.

Magnetic resonance imaging (MRI) can reliably differentiate nodular fatty infiltration of the liver from metastatic disease, in equivocal cases. Sequences include a combination of in-phase and opposed-phase gradient-echo imaging, fat saturation techniques and ferumoxide-enhanced MRI.,

Splenic enlargement is commonly seen in patients with non-alcoholic fatty liver, and the recognition of this association may halt further attempts at evaluating the cause of the splenic enlargement. ${ }^{4}$

\section{References}

1. Haaga JR, Lanzieri CF. Computed Tomography and Magnetic Resonance Imaging of the Whole Body. St Louis, Missouri: Mosby-year Book, 1994: 956-959.

2. Kroncke TJ.Multifocal nodular fatty infiltration of the liver mimicking metastatic disease on CT: Imaging findings and diagnosis using $\mathrm{MR}$ imaging. Eur Radiol 2000; 10: 1095-1100.

3. Hirohashi S. Nondiffuse fatty change of the liver: Discerning pseudotumour on MR images enhanced with ferumoxide: Initial observations. Radiology 2000 November 217: 415-420.

4. Tsushima Y. Spleen enlargement in patients with non-alcoholic fatty liver, correlation between degree of fatty infiltration in liver and size of spleen. Dig Dis Sci 2000 45(1): 196-200.

\section{The Pocket Radiologist series}

Each title in the POCKET RADIOLOGIST series provides you with the 100 most important diagnoses in a particular radiological speciality. For each diagnosis, you'll find 2 crisply reproduced images, plus consistent, bulleted summaries of... Key Facts; Imaging Findings; Differential Diagnosis; Clinical Issues; Pathologic Features and References. This unique organisation makes it remarkably easy to accurately and rapidly confirm or rule out a diagnosis! And all of the POCKET RADIOLOGIST titles are available as soft cover, pocket-sized books or as Personal Digital Assistant (PDA) software to meet your preferred reference style!

\section{Features}

HIGHRESOLUTION IMAGES: Multimodality Imaging. Vibrant colour graphics. Crisp black and white images.

FACTS: Bulleted text. Most essential information.

IMAGING FINDINGS: General findings. Imaging details of each relevant modality. Standard and advanced imaging. Recommendations.

DIFFERENTIAL DIAGNOSIS: Differential list with brief descriptions. Useful guidance to alternatives.

PATHOLOGY FINDINGS: Gross, microscopic. Etiology, epidemiology. Genetics.

CLINICAL ISSUES: Presentation. Natural History. Prognosis.

Treatment options.

SELECTED REFERENCES: Radiologists and trainees.

\section{Titles in this exciting series}

Pocket Radiologist Paediatric: Top 100 Diagnoses

Coming May 2002, paperback book R850. PDA CD-Rom R1 095

Pocket Radiologist Cardiac Top 100 Diagnoses

Coming May 2002, paperback book R850. PDA CD-Rom R1 095

Pocket Radiologist Spine Top 100 Diagnoses

Coming May 2002. Paperback book R850. PDA CD-Rom R1 095

Pocket Radiologist Brain Top 100 Diagnoses

February 2002. Paperback book R850. PDA/CD-Rom R1 095

Pocket Radiologist: Head \& Neck Top 100 Diagnoses

February 2002. Paperback book R850. PDA/CD-Rom R1 095

Pocket Radiologist: Musculoskeletal Top 100 Diagnoses February 2002. Paperback book R850. PDA/CD-Rom R1 095

SA Medical Association, Private Bag X1, Pinelands 7430. Tel (021) 530-6527, Fax (021) 531-1426.

E-mail: fpalm@samedical.org Please allow 4 weeks for delivery. Prices subject to foreign exchange fluctuations. 